\title{
Efficient Channel Utilization Schemes for IEEE 802.11 DCF over MANET
}

\author{
NaOki Nakamura, ${ }^{\dagger 1}$ Debasish Chakraborty, ${ }^{\dagger 2}$ \\ Apichet Chayabejara, ${ }^{\dagger 3}$ Gen Kitagata,${ }^{\dagger 3}$ Takuo Suganuma, ${ }^{\dagger 3}$ \\ Goutam ChakrabortY ${ }^{\dagger 4}$ and Norio Shiratori ${ }^{\dagger 3}$
}

\begin{abstract}
The MAC protocol of IEEE 802.11 reduces hidden terminal problem using the RTS/CTS handshaking mechanism. However, it lacks the ability to release or reallocate a channel that was reserved but not used. For Mobile Ad hoc NETworks (MANET), this often keeps the channel unnecessarily inaccessible, resulting in inefficient channel utilization. In this paper, we propose schemes for releasing and reallocating unused channels in MANET deployed over IEEE 802.11. We introduce a Network Allocation Vector (NAV) updating method that can improve the performance of the existing RTS Validation scheme and our proposed Extra Frame Transmission scheme. We then combine these schemes as a further improvement. Through simulations, done in different scenarios with varying networking load and node density, we were able to show that combining these schemes leads to a throughput improvement of up to $40 \%$. In addition, our proposed mechanisms have no compatibility problems.
\end{abstract}

\section{Introduction}

Wireless technology is faced with the inherent problems of lower bandwidth, high delay, and link errors. It is also difficult to implement collision detection. All these issues need to be considered when designing access controls for wireless medium.

There has been a lot of research on developing a wireless medium access control (MAC) that efficiently shares limited resources between all stations ${ }^{1), 2)}$. At present, IEEE 802.11 MAC is clearly the most accepted and widely used wireless technology. In addition to CSMA/CA, IEEE 802.11 introduces the Request-To-Send/Clear-To-Send (RTS/CTS) handshaking mechanism and Virtual Carrier Sensing to further reduce the probability of collisions that can occur due to hidden terminal problems.

However, using IEEE 802.11 in MANET, exacerbates hidden and exposed terminal problems ${ }^{3)}$. The ultimate results are severe degradation of throughput and instability of networks. It has been shown that this problem

$\dagger 1$ Tohoku University School of Medicine

$\dagger 2$ National Institute of Information and Communications Technology Tohoku Research Center

†3 Graduate School of Information Sciences / Research Institute of Electrical Communication, Tohoku University

†4 Department of Software and Information Science, Iwate Prefectural University is more severe in large and dense ad hoc networks ${ }^{4)}$. So improvement of performance for IEEE 802.11 over MANET is an important issue.

In disaster rescue operations or on the battlefield, intensive communications among many nodes should occur frequently to enable monitoring of the situation, or contact with relatives, friends, or peers. This would raise the burst traffic from time to time, and the network would suffer from severe performance degradation due to the false blocking problem ${ }^{5)}$.

A false blocking problem unnecessarily prevents nodes from transmitting in a given instant ${ }^{5)}$. In the worst case, it can lead to a pseudo deadlock situation when all neighboring nodes may become blocked and unable to transmit frames for long periods of time. This happens when an RTS frame reserves the channel even though the channel remains unused. Ray, et al. ${ }^{5)}$ proposed RTS Validation, where a channel is released when each node assumes that CTS is missing after it receives an RTS frame, based on physical carrier sensing.

With the same motivation we proposed a scheme called "Extra Frame Transmission" to manipulate frame transmission during RTS/CTS handshaking ${ }^{6)}$. When CTS is not received for some specific duration after a node sends an RTS frame, it will subsequently send another small frame to other destinations. Both these schemes ${ }^{5), 6)}$ reuse the channel that is unnecessarily reserved. The main differ- 
ence between RTS Validation scheme ${ }^{5)}$ and Extra Frame Transmission scheme ${ }^{6}$ ) is the node responsible for detecting the interruption of RTS/CTS handshaking. In our method ${ }^{6)}$ the sender node is responsible for interruption detection, whereas in Ray's ${ }^{5)}$, neighboring nodes are responsible.

Our aim is to further improve our scheme ${ }^{6}$ ) for releasing and reusing unused channels by minimizing wasted channel as much as possible. To make sure that happens, we introduce another extra frame called "Reverse Extra Frame". We also note that there is scope for improvement when channel release schemes are not applicable. We have shown that a "NAV updating" scheme can improve the performance of both RTS Validation and Extra Frame Transmission. In addition, because these schemes can work independently, we combined them. Moreover, our proposed solutions are compatible with standard IEEE 802.11, and therefore can be easily deployed. Results from simulations verify the effectiveness of our combination of schemes. Our schemes work well, especially when node density is high. This is because high node density increases the probability of false blocking, making our schemes' effects more prominent. We observed that our proposed scheme was able to increase throughput by up to $40 \%$ over that of standard IEEE 802.11.

The rest of this paper is organized as follows. In Section 2, we summarize the related work. Basic operations of IEEE 802.11 and its effect on MANET during RTS/CTS failure are explained in Section 3. Our proposed schemes to enhance IEEE 802.11 performance are explained in Section 4. The effectiveness and evaluation of the proposed schemes are discussed in Section 5. Finally we conclude our work in Section 6.

\section{Related Work}

RTS Validation has been proposed to mitigate the false blocking problem where the nodes that have received RTS inhibit themselves from transmitting in the chain ${ }^{5)}$. Upon overhearing an RTS frame, nodes listen to the medium whether the corresponding DATA frame transmission has taken place or not. They do this based on physical carrier sensing. If transmission has not taken place, the medium should have remained idle for an expected duration. At this point nodes start to overhear the DATA frame transmission since they have received an RTS frame. When the medium remains idle for the specified duration since the node received an RTS frame, it will conclude that an interruption of RTS/CTS handshaking has occurred. Then the node will release the NAV registered by that RTS frame and stop deferring. Subsequently, each node releases the channel independently.

Harada, et al. ${ }^{7}$ ) introduced a new frame, called Cancel RTS (CRTS), which left the reserved channel free in order to decrease degradation of channel utilization caused by failure to obtain a channel during RTS/CTS handshaking. In this scheme, when a sender node does not receive CTS correctly for its RTS, it sends a CRTS frame. Then, neighboring nodes, upon overhearing CRTS, cancel the NAV set by the RTS. However, the introduction of a new frame can cause compatibility problems with standard IEEE 802.11.

\section{Background}

\subsection{Basic Access Mechanism}

IEEE 802.11 DCF is based on the carrier sense multiple access (CSMA) technique ${ }^{8)}$. Before initiating a transmission, a station senses from the medium whether another station is transmitting or not. If the medium is sensed to be free for a DCF Interframe Space (DIFS) के interval, the transmission may proceed. On the other hand, if the medium is busy, the station must defer its transmission until the end of the current transmission. Then, it will wait for an additional DIFS interval and generate a random backoff timer before transmission. The counter is decreased as long as the medium is sensed as idle. It is frozen if the medium becomes busy and then resumed when the medium is again sensed as idle for a duration longer than a DIFS interval. Only when the backoff counter reaches zero, the station can transmit its frames.

\subsection{RTS/CTS Handshaking Mecha-} nism and Virtual Carrier Sensing

The RTS/CTS access method is provided in IEEE 802.11 as an option for reducing the collisions caused by hidden terminal problems. When a station needs to transmit a data frame longer than the RTS-Threshold, it

\footnotetext{
F Four types of interframe space have been specified in IEEE 802.11 to prioritize different accesses: Short IFS (SIFS) for highest priority, followed by DCF IFS (DIFS), PCF IFS (PIFS), and Extended IFS (EIFS).
} 


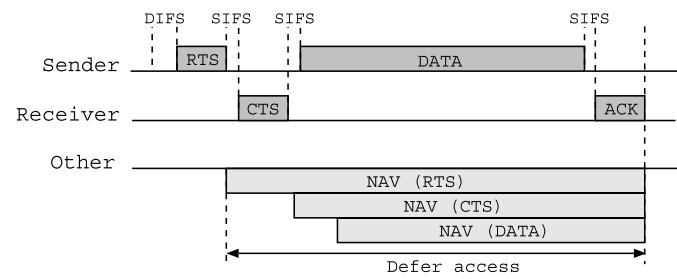

Fig. 1 The RTS/CTS access mechanism.

follows the backoff procedure as in the basic mechanism described before. After that, instead of sending a data frame, it sends a special short control frame called a Request-To-Send (RTS). This frame includes information about the source, destination, and duration required by the following transactions (CTS, DATA, and ACK transmission). Upon receiving the RTS, the destination responds with another control frame called a Clear-To-Send (CTS), which also contains the same information. The transmitting station is allowed to transmit data if the CTS frame is received correctly.

All other nodes overhearing either RTS and/or CTS frames adjust their Network Allocation Vector (NAV) to the duration specified in the RTS/CTS frames, as illustrated in Fig. 1. The NAV contains the duration for which the channel will be unavailable and is used as virtual carrier sensing. Stations defer transmissions if either physical or virtual sensing finds that the channel is busy. Nevertheless, if a receiver's NAV is set while the data frame is received, DCF allows the receiver to send the ACK frame.

Bianchi ${ }^{9)}$ examined the early collision detection ability of the RTS/CTS mechanism in the absence of CTS. An absence of CTS implies a collision has occurred, and with this information, collision can be detected early. However, the protocol cannot free or reallocate a channel that was already reserved by the RTS frame. Stations receiving only the RTS frame but not CTS cannot assume that the transmission is not taking place. Therefore, they defer the channel access for an interval declared in the last RTS. This results in a waste of channel capacity around the sender node.

\subsection{RTS/CTS Induced False Blocking}

In this section, we analyze situations in which the CTS is not received by the sender and discuss how to improve channel utilization in different situations.

Situation 1: Backoff timers at two or more stations reach zero at the same time and

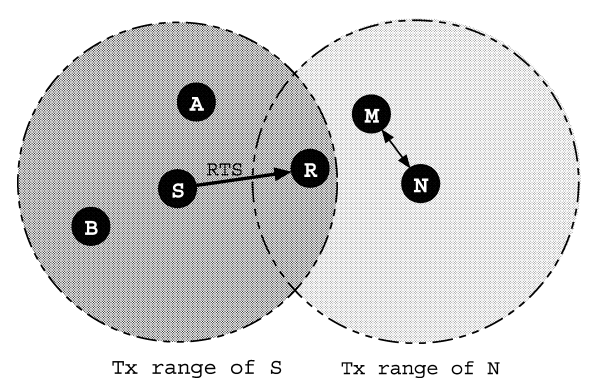

Fig. 2 Illustration of situation 2.

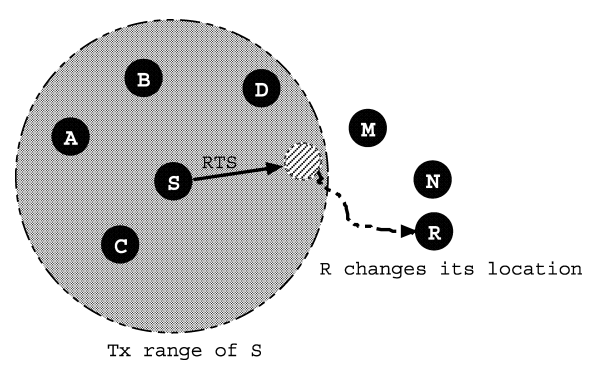

Fig. 3 Illustration of situation 3 .

send the RTS frame simultaneously, so the sender does not receive the CTS frame. This happens more frequently as network traffic increases.

Situation 2: As is illustrated in Fig. 2, station $S$ starts the RTS/CTS sequence while another transmission, which interferes with the reception but is out of $S$ 's sensing range, is been carrying on, say, between $\mathrm{N}$ and M. Even if the RTS correctly reaches the receiver, the virtual carrier sensing at station $R$ will forbid the CTS response.

Situation 3: This situation occurs when the intended receiver $R$, moves to a new position that is out of communication range of $S$, as shown in Fig. 3. Hence, it cannot receive RTS from $S$.

Since stations are free to move arbitrarily, the above situations occur regularly in MANET, where stations route packets through each other in multi-hop fashion. In wireless networks, only a single node is allowed to transmit at a particular time, and many nodes around the sender are blocked. Neighbors of a blocked node are unaware of this blocking, so a node may initiate communication with a node that is presently blocked. Because it is blocked, the destination is not able to respond to the RTS frame. However, the sender interprets this as channel contention and enters backoff. Its neighboring nodes are prevented from decrementing 
the backoff counter and from sending frames because of the NAV set by RTS.

This false blocking takes place because all the nodes that receive RTS inhibit themselves from transmitting. This problem can become severe when it occurs in a circular fashion and creates pseudo deadlock ${ }^{5)}$. This unnecessary blocking leads to lower channel utilization and route failure. Therefore, to increase channel efficiency and throughput, releasing the unused channel is an imperative issue. RTS Validation mitigates the above problem, but there is still wasted channel capacity.

In the next section, we explain our proposed schemes and our method of maximally reusing wasted channel capacity while maintaining compatibility with IEEE 802.11.

\section{Enhancement of Channel Utiliza- tion}

We propose four different schemes for more efficient use of channel capacity in MANET. In this section we describe the schemes. They are, (i) NAV updating, (ii) Extra Frame Transmission (EFT), (iii) combinations of schemes, and (iv) Reverse Extra Frame Transmission (REFT) and are discussed in Section 4.1, 4.2, 4.3, and 4.4 respectively.

\subsection{NAV Updating}

In the RTS Validation mechanism ${ }^{5)}$, the node cannot reset the NAV if the NAV has already been set by other RTS frames. As NAV cannot be restored to it's previous value, RTS Validation may not always be applicable. As network traffic increases, RTS Validation becomes less applicable. As a result, RTS Validation cannot fully restore unused channels. The scheme proposed in Ref. 10), applying RTS Validation, can be improved by adjusting NAV to a more appropriate value. However, due to its high dependence on RTS Validation, it is difficult to further improve this scheme by combining it with other methods.

Because of this, we modify the NAV operations as follows:

(i) We divide the original NAV into two parts: one is a set of $\mathrm{NAV}_{\mathrm{k}}$ indexed with the corresponding node-ID, and the other is NAV other

(ii) NAV used for the operation of virtual carrier sense is calculated by the maximum value in the sets of $\mathrm{NAV}_{\mathrm{k}}$ and $\mathrm{NAV}_{\text {other }}$.

(iii) $\mathrm{NAV}_{\mathrm{k}}$ is adjusted when it overhears RTS or DATA frames from node , $_{\text {, and }} \mathrm{NAV}_{\text {other }}$

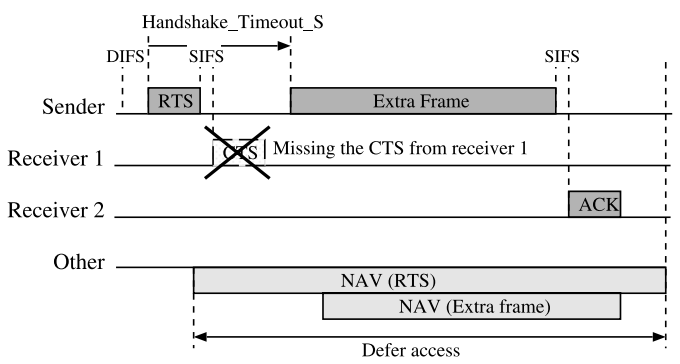

Fig. 4 Extra frame transmission.

is adjusted by cases other than the RTS/DATA frame, as when it is receiving a CTS frame or suffering from a collision.

(iv) Override $\mathrm{NAV}_{\mathrm{k}}$ by the duration specified by the RTS/DATA frame, irrespective of its length of the duration. This means that when a node overhears an RTS/DATA frame of node $\mathrm{k}_{\mathrm{k}}$, then $\mathrm{NAV}_{\mathrm{k}}$ will be updated by the duration specified in the frame.

(v) If needed, the RTS Validation scheme with NAV updating will reset $\mathrm{NAV}_{\mathrm{k}}$, whereas the original RTS Validation scheme merely resets the NAV that was set by RTS.

Our proposed NAV updating scheme can handle NAV for multiple nodes with the senders' ID corresponding to an RTS/CTS handshaking failure, making cancellation of the NAV more flexible and convenient. This scheme helps RTS Validation cancel the NAV and improve channel utilization.

\subsection{Extra Frame Transmission (EFT)}

Extra Frame Transmission works as shown in Fig. 4. Here, by extra frame, we mean transmission of another extra frame as a replacement for the frame originally intended to be transmitted. After the sender has transmitted RTS for receiver 1 and waited until it decides that CTS is not going to come, it picks a frame from the sending queue and immediately transmits it to the alternate receiver, if an appropriate one exists. Here, the sender skips the RTS/CTS handshaking step. The extra frame will be removed from the queue if the transmission is completed (confirmed by ACK from receiver) ${ }^{8)}$ or if the transmitted extra frame is a broadcast type. Regardless of the success of the extra frame transmission, the sender goes back to normal operation by scheduling the retransmission of the original frame with the Contention Window (CW) doubled.

Since the standard protocol does not specify a timeout value for CTS response, the sender normally stays idle until the end of the allo- 
cated duration. However, the RTS/CTS sequence uses strict timing, so we introduce a new parameter, Handshake_Timeout_S defined in Section 5.3, which specifies the maximum time required to receive a CTS. Having waited for this Handshake_Timeout_S, the sender is assured that the CTS response from the receiver will not come at all.

The transmitted replacement frame, should satisfy all the following properties:

(1) The extra frame should be destined for a station other than the currently intended one. Since no reply was received from the current destination, any further attempt to the same station would be futile.

(2) The selected extra frame should be either a broadcast frame or unicast frame smaller than RTS-Threshold. Thus, the frame can be immediately transmitted without following RTS/CTS frame exchange protocol.

(3) The chosen extra frame should be the first in the queue destined for a particular receiver. For example, if there are two frames for the same destination at the sender, and the first frame is larger than RTS-Threshold, but the second frame is not, then none of the second frame will be sent, even though second frame may satisfy the first two conditions. This is to avoid out-of-order transmission.

It is natural for the probability of false blocking to increase as traffic load and node density increase. When this happens, there is a fair chance of a successful transmission of this extra frame because the channel around the sender has already been reserved by a previous RTS frame. Thus, we can deliver an extra frame that cannot be sent following normal operation.

With the NAV updating schemes, introduced earlier, there is a two fold benefit from this scheme. The nodes that received the RTS and blocked their channels will be allowed to cancel the original NAV duration.

In fact, the nodes will readjust the previously set NAV with the duration of new extra frame. Because of the way we selected the extra frame, NAV should have a shorter value in the duration field than the current NAV for the RTS sender. When NAV updating is used, if the selected extra frame is a broadcast frame (for whom NAV duration is zero) the overhearing nodes can re-set the NAV value for RTS and completely cancel the NAV.

\subsection{Combination of RTS Validation and Extra Frame Transmission}

Extra Frame Transmission and RTS Validation ${ }^{5)}$ can work independently on the sender node and neighboring nodes. To further improve performance, we propose an approach that combines RTS Validation and Extra Frame Transmission.

Since an appropriate extra frame may not always be available in the sender node's waiting queue, channel reuse is not possible as frequently as with RTS Validation. Thus Extra Frame Transmission is works by delivering an extra frame whenever possible, whereas RTS Validation works by releasing channels. To enable them to work together in parallel, we define two parameters, Handshake_Timeout_N, Handshake_Timeout_S as follows:

\section{Handshake_Timeout_S :}

RTS_Tx_time + propagation_delay + SIFS+

CTS_Tx_time + propagation_delay

\section{Handshake_Timeout_N :}

propagation_delay + SIFS + CTS_Tx_time+

propagation_delay + SIFS +

propagation_delay + SIFS

Where, Tx_time represents the transmission time.

With the timeouts defined above, when a sender node detects an interruption of RTS/CTS handshaking, it sends an extra frame if one is available. The extra frame will deliver data and release the channel by virtue of updating the NAV. Even if there are no extra frames, RTS Validation will reset the NAV. Thus, the combination of schemes effectively improves channel utilization.

\subsection{Reverse Extra Frame Transmis- sion (R-EFT)}

Another way of aggressively reusing channels is by introducing a new type of extra frame called "Reverse Extra Frame". Since the frame transmission timing is the same as when channels are released by RTS Validation, it can be described as a subset of RTS Validation. The algorithm for combining the schemes, including Reverse Extra Frame, is shown in Fig. 5.

The idea stems from the fact that, generally speaking, once RTS frame has been sent, the sender's surrounding area (transmission range) is relatively free from collision for the duration specified in the RTS frame. If one of the neighboring nodes needs to send a frame to the sender, that frame is expected to reach it 


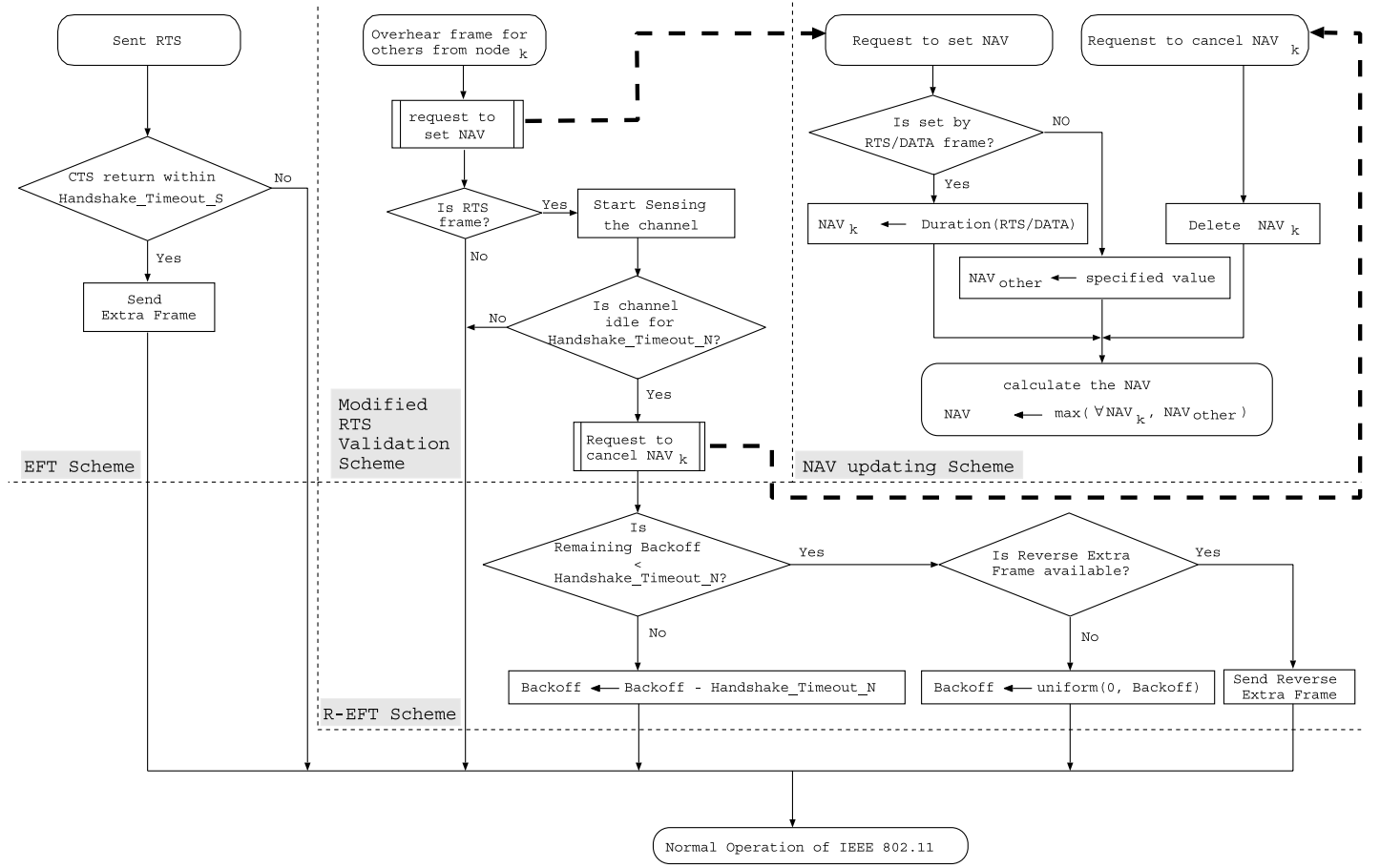

Fig. 5 Flow chart of combination of schemes.

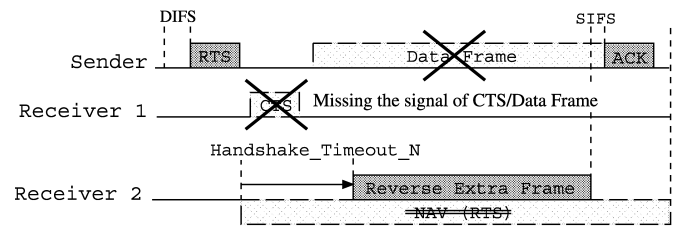

Fig. 6 Reverse extra frame transmission.

successfully. To exploit this relatively safe period of time to reuse the channel, we allow the neighboring nodes to send an extra frame, if there is any, to the node that originated the RTS. Reverse Extra Frame Transmission works as shown in Fig. 6.

Collision prevention is not integrated into the Reverse Extra Frames scheme. Collisions are still possible as there is more than one eligible candidate for sending an extra frame. To reduce the probability of collision, the following constraints are introduced:

- Reverse Extra Frame should be the first frame in the queue, and should be destined for the node which sent the RTS frame.

- The duration of Reverse Extra Frame should be smaller than that specified in RTS.

- The node should have a short backoff timer that expires if the node does not receive the RTS frame.
If an appropriate reverse extra frame is found, it is sent immediately, and is removed from the queue after successful transmission (confirmed by ACK from the sender). The node that transmitted the Reverse Extra Frame goes back to normal operation.

We have seen that when RTS/CTS handshaking is interrupted, the neighboring nodes will be inhibited from transmitting. RTS Validation can release the channel, but the nodes cannot recover from the loss incurred by the interruption. This is because, when the nodes sensed the channel as busy, their backoff timers were halted and stopped decrementing during RTS/CTS handshaking.

When Reverse Extra Frame is available, its transmission improves channel utilization. However, due to restrictions imposed to prevent collision, transmission of the Reverse Extra Frame may not always be possible. When there is no Reverse Extra Frame, we allow the nodes to decrement their respective backoff timers.

Basically we allow those nodes to decrement the time equal to the Handshake_Timeout_N, from their respective remaining backoff timers. For those nodes whose remaining backoff timer time is less than or equal to the Handshake_Timeout_N, a value between 
(0, current backoff time) is chosen with uniform probability to differentiate their access to collision avoidance.

Thus, when Reverse Extra Frame is not available, the nodes will decrease duration of their backoff timer. We can thus reduce the waiting time for nodes for their next transmission, and increase throughput.

\subsection{Compatibility with IEEE 802.11}

All frame formats used in our schemes are IEEE 802.11 compatible. In addition, after the node releases the NAV, nodes behave according to IEEE 802.11 DCF operation. We are not incorporating any extra frames like CRTS $^{7)}$. Thus our proposed scheme is compatible with the existing IEEE 802.11 standard. Even stations that do not implement the proposed schemes will work as well.

\section{Performance Evaluation and Dis- cussions}

\subsection{Simulation Scenario}

The most widely used network simulator, $n s$ $2^{11), 12)}$ was used to evaluate the effectiveness of our mechanism. We compared the performance of standard IEEE $802.11^{8)}$, RTS Validation ${ }^{5)}$, and our proposed schemes.

The network model of multi-hop wireless topology, and routing protocol ${ }^{13), 14)}$ AODV (Ad hoc On demand Distance Vector) is used. The link layer is a shared radio medium with a nominal channel bit rate of $1 \mathrm{Mbps}$. The antenna is omni-directional with a radio range of 250 meters. Set up parameters are listed here: slot time $=20 \mu \mathrm{sec}$, SIFS $=10 \mu \mathrm{sec}$, DIFS $=$ $50 \mu \mathrm{sec}$, propagation delay $=2 \mu \mathrm{sec}$, RTSThreshold $=0$ bytes, queue size $=50$ frames.

Traffic source and destination pairs are randomly spread over the network. The type of traffic is constant bit rate (CBR) with packet size randomly chosen from the range of 5122048 bytes. We have created 30 sets of CBR traffic with the same transmission rate. The sum of all senders' transmission rates is considered to be offered load. For example, if the offered load is $600 \mathrm{kbps}$, then the transmission rate of each set of CBR traffic is $20 \mathrm{kbps}$.

The Random Waypoint Mobility Model ${ }^{15}$ ) is used. In this model, a mobile station begins by staying in one location for a certain period of time (we call this pause_time). Once this time expires, this station chooses a random location in the simulation field and moves to that location with a velocity between

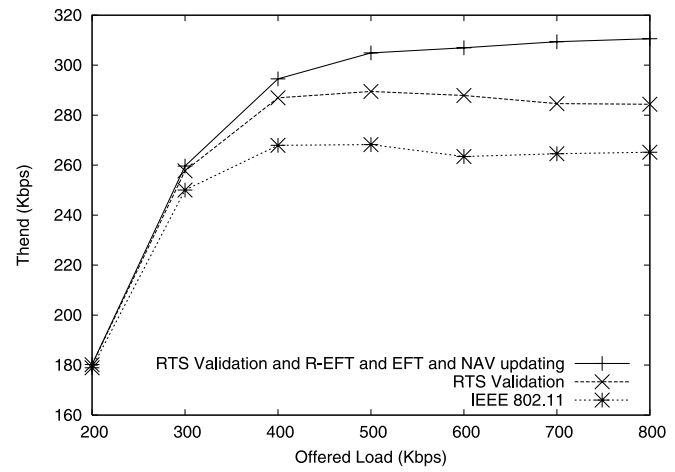

Fig. 7 End to end throughput as a function of offered load (number of nodes is fixed at 50).

[min_speed, max_speed], which is randomly selected with uniform probability. And each node moves according to Random Waypoint Model with parameters max_speed $=10(\mathrm{~m} / \mathrm{sec})$, min_speed $=0(\mathrm{~m} / \mathrm{sec})$, pause_time $=50(\mathrm{sec})$.

Each simulation was run on a $1500 \times 500 \mathrm{~m}^{2}$ area for 700 seconds. We started by measuring the performance parameters from the 100th second until the end of the simulation, i.e., for a duration of 600 seconds. These graphs show the average of 50 simulation results.

\subsection{Results and Analysis}

In this section we compare the performance our proposed schemes, IEEE 802.11, and RTS Validation ${ }^{5)}$. We consider two different scenarios. In one, the number of stations is fixed at 50 , and we varied the offered load between $200 \mathrm{kbps}$ and $800 \mathrm{kbps}$. In the other, fixing the offered load at $450 \mathrm{kbps}$ where the interruption of RTS/CTS handshaking occurs relatively frequently, we varied the number of nodes in the network from 10 to 90 .

First we evaluated the effects of the proposed combination of schemes in terms of end-to-end throughput $\left(T h_{\text {end }}\right)$ in the above two scenarios. Suppose, $U_{i}$ is the amount of CBR data (in bits) successfully received during the period of $t$ sec (In this simulation, as mentioned in Section 5.1, we chose $t$ as 600). Then $T h_{\text {end }}$ is defined as:

$$
T h_{\text {end }}=\frac{\sum_{i=1}^{N} U_{i}}{t}
$$

Figures $\mathbf{7}$ and $\mathbf{8}$ show the end-to-end throughput for IEEE 802.11, RTS Validation and the proposed scheme for two different scenarios.

Figure 7 shows $T h_{\text {end }}$ of various schemes with 


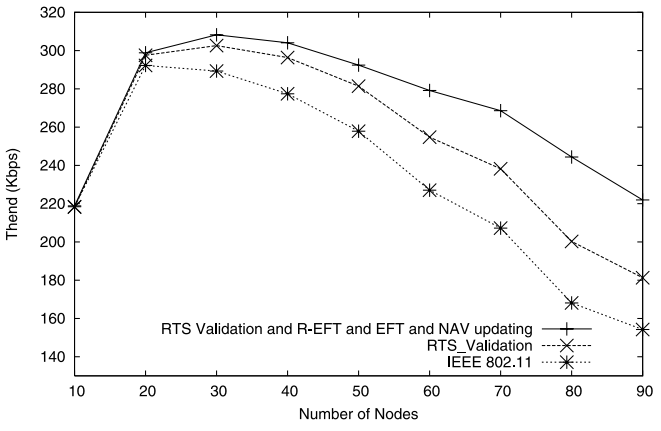

Fig. 8 End to end throughput as a function of node density (total offered traffic is fixed at $450 \mathrm{kbps})$.

respect to network traffic. When the offered load is low, all the schemes show very similar throughput. In this case, channel reuse schemes will not have a significant effect because of RTS/CTS handshaking is not interrupted very frequently. As traffic increases, our proposed scheme achieves the highest throughput due to its channel reuse effect.

As shown in Fig. 8, when the number of nodes is small, $T h_{\text {end }}$ is low due to the partitions in the networks. Then, as the number of nodes increases, interference increases. As a result, the interruption of RTS/CTS handshaking occurs more frequently. When node concentration is high, false blocking becomes severe, leading to performance degradation. Even in these conditions, our scheme can improve performance by up to about $40 \%$ compared to standard IEEE 802.11.

To analyze the performance of our scheme and give a clear idea of the effectiveness of different schemes, we compare throughput as a function of offered load in the MAC layer for six different combinations of schemes in Fig. 9. MAC layer throughput $\left(T h_{m a c}\right)$ is defined as:

$$
T h_{m a c}=\frac{\sum_{i=1}^{N} u_{i}}{t}
$$

where $u_{i}$ is the amount of data frame in bits successfully transmitted by node $i$ for $t$ sec.

From Fig. 9, we can draw the following conclusions.

The NAV updating scheme improves the throughput of Extra Frame and RTS Validation by increasing channel release. In regard to the channel release effect of EFT and REFT, it is seen that "RTS Validation with NAV

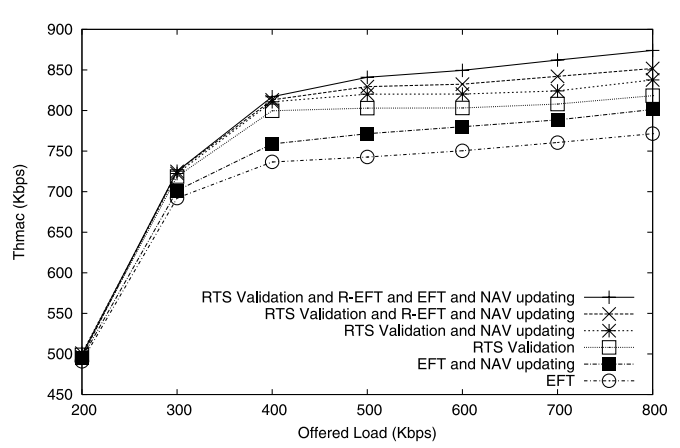

Fig. 9 MAC layer throughput comparison as a function of offered load (number of nodes is fixed at $50)$.

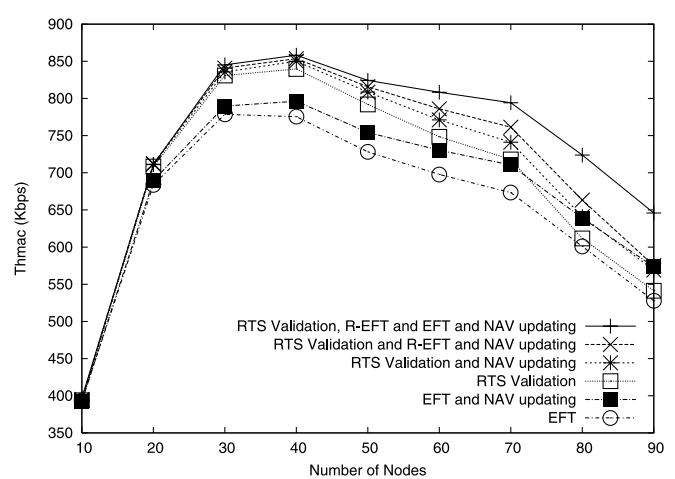

Fig. 10 MAC layer throughput comparison as a function of node density (offered load is fixed at $450 \mathrm{kbps})$.

updating" is more effective than "Extra Frame with NAV updating". This is because the RTS Validation scheme is executed more frequently than that of Extra Frames Transmission. Besides that, if we gradually add NAV updating, R-EFT, and EFT to RTS validation, the combinations of schemes also gradually improve performance. As observed above, the combination of all the schemes works well in both scenarios.

On the other hand, when the number of nodes is varied, as in Fig. 10, RTS Validation, including its variants (RTS Validation with NAV updating and Reverse Extra Frame), throughput decreases rapidly as the number of nodes increases. In the worst case, the performance of RTS Validation is worse than that of Extra Frame with NAV updating.

This is because RTS Validation and its variants detect the interruption of RTS/CTS handshaking if no signal is sensed by physical carrier sensing. As the number of nodes increases, frames will more often be reached. This interferes with interruption detection resulting 


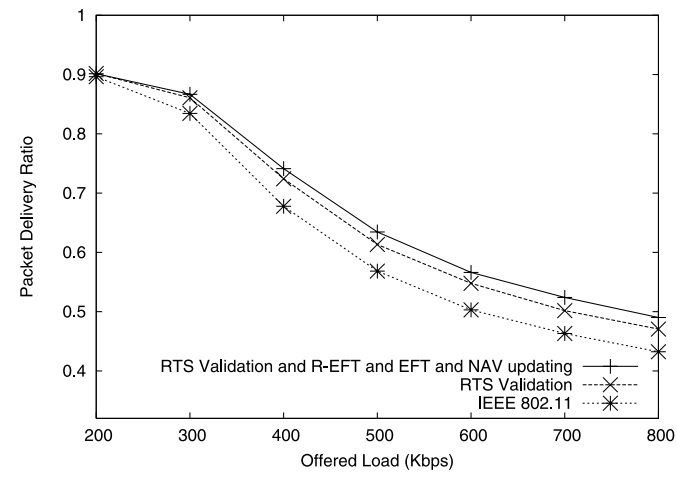

Fig. 11 Packet delivery ratio as a function of offered load (number of nodes is fixed at 50).

in performance degradation. While the EFT scheme is invoked when a CTS frame is missing, it is unrelated to the physical carrier sensing used by RTS validation. Therefore, schemes combined with EFT can keep the improvement level high due to their two-fold advantage of sending extra frames and releasing the channel even when the number of nodes is large. The combination of EFT, RTS Validation, and R-EFT with NAV updating performs steadily even as the number of nodes increases. The improvement is more remarkable when the number of nodes is 60 or greater.

Therefore, the combination of RTS Validation and its variants with Extra Frame works in a complementary way. This leads to higher performance in both scenarios.

We also tested whether our combined scheme causes any transmission reliability degradation by introducing schemes for reusing and releasing the unused channel as aggressively as possible by measuring the delivery rate of CBR packets.

Packet Delivery ratio (PD) is computed as:

$$
P D=\frac{\sum_{i=1}^{i=N} R_{i}}{\sum_{i=1}^{i=N} S_{i}}
$$

$S_{i}$ means total data size of CBR packet node $i$ sent, $R_{i}$ means total data size of CBR Packet node $i$ received.

Figures 11 and 12 show CBR packet delivery ratio as a function of offered traffic load and node density respectively, for RTS Validation and IEEE 802.11 and our combined scheme.

In Fig. 12, the packet delivery ratio is smallest when there are ten nodes. This is because there

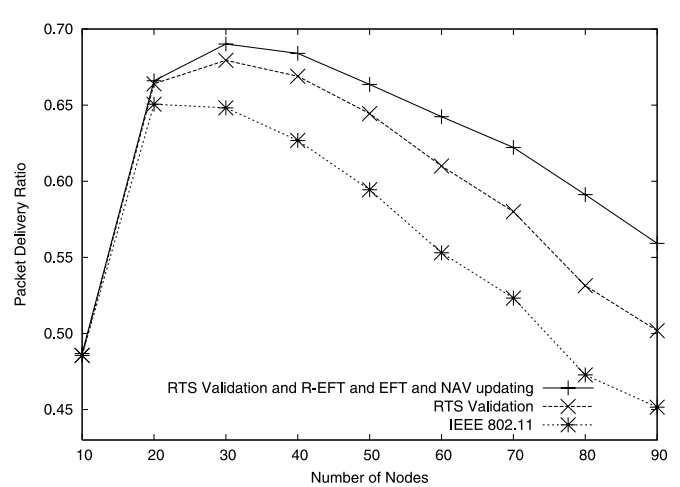

Fig. 12 Packet delivery ratio as a function of node density (offered load is fixed $450 \mathrm{kbps}$ ).

are partitions in the networks so that packets cannot reach the destination. As the number of nodes increases, the packet delivery ratio peaks due to reduction in the size of the partitions.

In both scenarios, when RTS/CTS is not interrupted frequently, all schemes have similar packet delivery ratios. As the interruption of RTS/CTS handshaking becomes more frequent, our combined scheme yields a higher packet delivery ratio than the other schemes in both scenarios. In the high node density scenario, especially when interruption of RTS/CTS handshaking occurs more frequently, our scheme's packet delivery ratio is higher than those of other schemes. This is because reusing the wasted channel aggressively enables nodes to deliver more packets in a stable manner, consequently increasing the packet delivery ratio. These results show that regardless of whether channels are released/reused aggressively, our combination of schemes does not adversely effect packet delivery ratio.

\section{Conclusion}

In this paper we have shown that our proposed schemes can overcome the inherent channel inefficiency of RTS/CTS handshaking in mobile ad hoc networks, especially when node density is high and interruption is frequent. At the same time, our enhancements do not suffer from any compatibility problems and generate no additional overhead, which is vital for smooth deployment.

Results obtained from extensive simulations show that our combined method considerably improves throughput compared to standard IEEE 802.11 and the RTS Validation scheme. Especially when interruption of RTS/CTS handshaking occurs more frequently, our com- 
bined scheme was able to achieve more than $40 \%$ gain in throughput compared to IEEE 802.11 .

We would like to investigate our schemes using different routing protocols and with varying node velocity. Distribution of nodes with and without our proposed schemes could be interesting and might further improve channel utilization. We plan to analyze these effects in our future research.

\section{References}

1) Bharghavan, V., Demers, A., Shenker, S. and Zhang, L.: MACAW: A Media Protocol for Wireless LAN's, ACM SIGCOMM'94, pp.212225 (1994).

2) Karn, P.: MACA - A New Channel Access Method for Packet Radio, ARRL/CRRL Amature Radio 9th Computer Networking Conference, pp.134-140 (1990).

3) $\mathrm{Xu}, \mathrm{S}$. and Saadawi, T.: Does the IEEE 802.11 MAC Protocol Work Well in Multihop Wireless Ad Hoc Networks?, IEEE Communication Magazine, Vol.39, No.6, pp.130-137 (2001).

4) Wang, Y. and Garcia-Luna-Aceves, J.J.: Collision Avoidance in Multihop Ad Hoc Networks, Proc. IEEE/ACM MASCOT'O2, pp.145-154 (2002).

5) Ray, S., Carruthers, J.B. and Starobinski, D.: RTS/CTS-induced congestion in ad-hoc wireless LANs, Proc. IEEE WCNC 2003, pp.15161521 (2003).

6) Chayabejara, A., Zabir, S.M.S. and Shiratori, N.: An Enhancement of IEEE 802.11 for Efficient Operations in MANET, Proc. IASTED Parallel and Distributed Computing and Systems, pp.200-206 (2003).

7) Harada, T., Ohta, C. and Morii, M.: Improvement of TCP throughput for IEEE 802.11 DCF in wireless multi-hop networks, IEICE Trans. Comm., Vol.J85-B, No.12, pp.2198-2208 (2002).

8) IEEE Standard Association: IEEE standard for wireless LAN medium access control (MAC) and physical layer (PHY) specification [Online]. Available: http://standards.ieee.org/ getieee802/802.11.html (1999).

9) Bianchi, G.: Performance Analysis of the IEEE 802.11 Distributed Coordination Function, IEEE Journal on Selected Areas In Communications, Vol.18, No.3, pp.535-547 (2000).

10) Yatabe, K., Uehara, H. and Yokoyama, M.: Solutions to NAV Blocking Problem Caused by RTS/CTS Mechanism in Wireless Multihop Networks, IEICE Technical Report:RCS2004127, Vol.104, No.257, pp.43-48 (2004).
11) The VINT Project: The Network Simulator Version 2 (ns-2). [Online]. Available: http://www.isi.edu/nsnam/ns/

12) Fall, K. and Varadhan, K.: The ns Manual. [Online]. Available: http://www.isi.edu/ nsnam/ns/ns-documentation (2002).

13) Perkins, C. and Belding-Royer, E.: Ad hoc On-Demand Distance Vector Routing, Proc. 2nd IEEE WMCSA'99, New Orleans, LA, pp.90-100 (1999).

14) Perkins, C., Belding-Royer, E. and Das, S.: Ad hoc On-Demand Distance Vector (AODV) Routing, RFC 3561 (2003).

15) Camp, T., Boleng, J. and Davies, V.: A Survey of Mobility Models for Ad Hoc Network Research, Wireless Communication and Mobile Computing (WCMC): Special issue on Mobile Ad Hoc Networking: Research, Trends and Applications, Vol.2, No.5, pp.483-502 (2002).

(Received May 19, 2006)

(Accepted November 2, 2006)

(Released February 7, 2007)

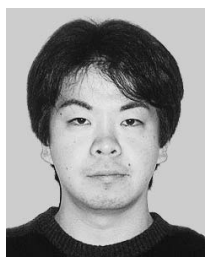

Naoki Nakamura is a research associate at Tohoku University School of Medicine, Japan. He received his MS in Information Engineering from Tohoku University in 2003. His research interests include wireless networking, distributed algorithms, and network performance evaluation. He is a member of IPSJ.

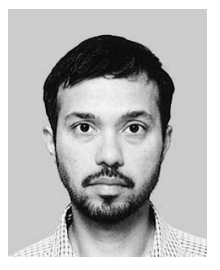

Debasish Chakraborty received his doctoral degree from the Graduate School of Information Science, Tohoku University, Japan in 1999. Previously he was a Telecommunication Advancement (TAO) research fellow and is now working as a National Institute of Information and Communication Technology (NiCT) foreign researcher at Tohoku Research Center. His main research interests are multicast routing algorithm, QoS, Internet traffic analysis, and wireless and ad hoc networking. 


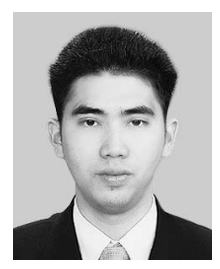

Apichet Chayabejara received his M.S. degree from Tohoku University, Japan in 2004. He was a technical consultant at Trend Micro (Thailand) before joining Cisco Systems (Thailand) in 2006 as a system engineer. His specialization is in the field of network security and wireless communication.

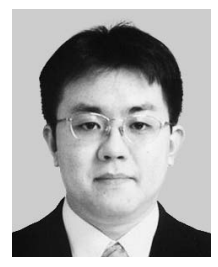

Gen Kitagata is a research associate of Research Institute of Electrical Communication of Tohoku University. He received his Dr. Eng. degree in information engineering from Tohoku University, Japan in 2002. His research interests include agent-based computing and network middleware design. He is a member of IPSJ and IEICE.

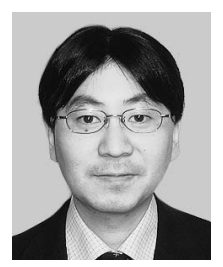

Takuo Suganuma is an associate professor of Research Institute of Electrical Communication of Tohoku University. He received his Dr.Eng. degree from Chiba Institute of Technology. His research interests include agent-based computing, flexible networks, and symbiotic computing. He is a member of IEEE, IEICE, and IPSJ.

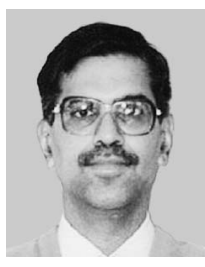

Goutam Chakraborty received his Ph.D. in 1993 from Tohoku University, Japan. Presently he is Professor and head of the Intelligent Informatics Laboratory, Department of Software and Information Science, Iwate Prefectural University, Japan. His main research interests are Soft Computing algorithms to solve pattern recognition, prediction, scheduling and optimization problems including applications in wired, and wireless networking problems.

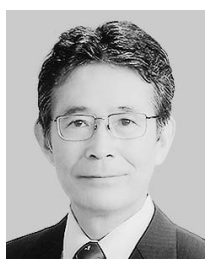

Norio Shiratori received his doctoral degree from Tohoku University, Japan in 1977. Presently he is a professor and vice director of Research Institute of Electrical Communication, Tohoku University. He has been engaged in research related to symbiotic computing paradigms between human and information technology. He was the recipient of IPSJ Memorial Prize Winning paper award in 1985, Telecommunication Advancement Foundation Incorporation award in 1991, best paper award of ICOIN-9 in 1994, IPSJ best paper award in 1997, and many others. He is a fellow of IEEE, IPSJ, and IEICE. 